\title{
Clinical Efficacy of Shenmai Injection in the Treatment of Cerebral Vasospasm after Ruptured Aneurysm Surgery
}

\author{
Tianya $\mathrm{Wu}^{{ }^{*}}$, Jingxin $\mathrm{Fu}^{1^{*}}$, Xinghuo Jin${ }^{1}$, Qichao Chen ${ }^{1}$, Huanming Huang², Shiqi Chen ${ }^{3}$, \\ Jun'an Zhou ${ }^{3}$, Longbiao $\mathrm{Xu}^{3 \#}$
}

${ }^{1}$ Department of Neurosurgery, Zhuji Affiliated Hospital of Shaoxing University, Zhuji People' Hospital of Zhejiang Province, Zhuji, China

${ }^{2}$ Department of Chinese Medicine, Zhuji Yaojiang Town Central Health Center of Zhejiang Province, Zhuji, China

${ }^{3}$ The Third Affiliated Hospital of Zhejiang University of Traditional Chinese Medicine, Zhuji, China

Email: ${ }^{* 18358569988 @ 163 . c o m ~}$

How to cite this paper: $\mathrm{Wu}$, T.Y., Fu, J.X., Jin, X.H., Chen, Q.C., Huang, H.M., Chen, S.Q., Zhou, J.A. and Xu, L.B. (2021) Clinical Efficacy of Shenmai Injection in the Treatment of Cerebral Vasospasm after Ruptured Aneurysm Surgery. Case Reports in Clinical Medicine, 10, 253-263.

https://doi.org/10.4236/crcm.2021.1010033

Received: September 7, 2021

Accepted: October 10, 2021

Published: October 13, 2021

Copyright $\odot 2021$ by author(s) and Scientific Research Publishing Inc. This work is licensed under the Creative Commons Attribution International License (CC BY 4.0).

http://creativecommons.org/licenses/by/4.0/

\begin{abstract}
Objective: To investigate the therapeutic effect of Shenmai Injection on postoperative cerebral vasospasm in patients with ruptured aneurysms. Methods: Seventy patients undergoing craniotomy for ruptured aneurysms in our hospital were selected as study subjects and randomly divided into control $(n=33)$ and research $(n=37)$ groups, they were treated with nimodipine and nimodipine combined with Shenmai injection after operation. The blood flow velocity in the middle cerebral artery (MCA) before and at 1,3, 7, 11 and 14 days after surgery and the incidence of cerebral vasospasm during these days were compared, and the GCS scores at 14 days postoperatively and GOS scores at 6 months postoperatively were compared between the two groups. Results: There were no statistically significant differences in the occurrence of cerebral vasospasm, GCS or GOS scores between the two groups $(P>0.05)$, but the period of postoperative cerebral vasospasm in the study group was significantly shorter than that in the control group. Conclusion: Shenmai injection has the effect of shortening the cycle of occurrence of cerebral vasospasm after the operation of ruptured aneurysms, promoting patients to recover as early as possible and reducing their physical and mental burden.
\end{abstract}

\section{Keywords}

Shenmai Injection, Nimodipine, Ruptured Aneurysm, Aneurysmal Subarachnoid Hemorrhage, Cerebral Vasospasm

*Tianya $\mathrm{Wu}$ and Jingxin Fu have equally contributed to this work as co-first authors.

${ }^{*}$ Corresponding author. 


\section{Introduction}

Subarachnoid hemorrhage (SAH) is an acute and severe cerebrovascular disease with an incidence of approximately 2-22/100,000 persons per year, of which about $85 \%$ is associated with spontaneous rupture of intracranial basilar artery aneurysms with a mortality rate as high as $35 \%, 1 / 3$ of survivors require lifelong care, and another 1/3 experience residual cognitive impairment [1] [2] [3] [4]. Cerebral vasospasm (CVS) is one of the serious complications after SAH with an incidence of $70 \%$. Cerebral ischemia induced by persistent constriction of intracranial arteries leads to neurological deficits in patients and is an important cause of aggravation and even death in patients after aneurysm surgery, which seriously compromises the curative effect [5] [6]. Therefore, it's important to prevent secondary cerebral vasospasm after the operation of ruptured aneurysms or shorten the duration of cerebral vasospasm for the purpose of promoting the postoperative rehabilitation of patients and improving their prognosis, but it's also one of the key and difficult points to be overcome in neurosurgery.

At present, a large number of studies have been conducted on the occurrence and development of CVS after the operation of ruptured aneurysms in the clinic, and the diagnosis, treatment and preventive measures of CVS have been partially clarified, but there are still some difficult and urgent problems to be solved. Nimodipine, a calcium ion channel antagonist, has been widely agreed on internationally for the clinical application in the prevention and treatment of CVS after ruptured aneurysms [7]. But the biggest side effect of nimodipine is that it can cause a decrease in arterial blood pressure, which will lead to a decrease in cerebral blood perfusion, and thus affects the functional recovery of nerve cells after ischemia, increasing the adverse outcome [8] [9]. Shenmai injection, derived from Shengmai San in Qianjin Yaofang, is a compound Chinese drug composed of red ginseng and Radix Ophiopogon japonicus, which has the effects of improving blood circulation and alleviating cell damage [10]. Because nimodipine alone still has limitations for preventing and treating CVS, in this study, we sought to observe the clinical efficacy of Shenmai injection for preventing CVS after treatment of ruptured aneurysms on the basis of maintaining the necessary dosage of nimodipine.

\section{Ethical Review}

This clinical research had met with approval of the clinical ethics committee of the Zhuji Affiliated Hospital of Shaoxing University. Written informed consent had been obtained from patients participating in the study.

\section{Materials and Methods}

\subsection{Subjects}

Patients after craniotomy for ruptured aneurysms who were hospitalized in the Department of Neurosurgery at our institution from January 2018 to March 2021 were selected for the study, and 70 eligible patients were included in this study 
strictly in accordance with the inclusion, exclusion, and shedding criteria. They were randomly divided into two groups according to the random number table method: 1) study group: 37 patients including 16 males and 21 females treated with nimodipine and Shenmai injection (mean age of $49.00 \pm 3.54$ years); 2) control group: 33 patients including 14 males and 19 females treated with nimodipine and glucose solution equivalent to dose of shenmai injection in study group (mean age of $47.70 \pm 3.23$ years). The gender, age distribution and preoperative GCS scores of the two groups were not significantly different $(P>0.05$, Table 1) and were comparable.

\subsection{Diagnosis Criteria}

\subsubsection{After Ruptured Aneurysm Surgery}

Patients were successfully clipped, contoured, or wrapped via craniotomy after confirmation of intracranial hemorrhage by $\mathrm{CT}$ and intracranial aneurysm diagnosis by DSA and/or CTA.

\subsubsection{Cerebral Vasospasm}

Patients after the operation of ruptured aneurysms can be diagnosed if they meet any two of the following criteria [11]: 1) fluctuating or progressive deterioration of consciousness status; 2) appearance or aggravation of focal localization signs and/or intracranial hypertension, ruling out water electrolyte acid-base balance disturbance; 3) cranial imaging (CT or MRI) suggested the presence of new cerebral infarct lesions, excluding intracerebral hemorrhage and hydrocephalus; 4) the mean blood flow velocity in the MCA on the affected side measured by transcranial Doppler ultrasound (TCD) was $>120 \mathrm{~cm} / \mathrm{s}$.

\subsection{Inclusive Criteria}

1) All cases were patients after surgery of ruptured aneurysms; 2) patients were graded I-IV by Hunt Hess and WFNS and 0 - 4 by modified Fisher; 3) had not been previously treated with other approaches for cerebral vasospasm; 4) patients themselves or their families volunteered to participate in this study.

\subsection{Exclusion Criteria}

1) Patients with severe systemic complications (severe dysfunction of important organs such as heart, liver, kidney, and lung), severe mental illness, and dementia;

Table 1. Comparison of study and control groups in basic condition.

\begin{tabular}{ccccc}
\hline Group & Total & Male & Age (years) & $\begin{array}{c}\text { preoperative GCS } \\
\text { scores }\end{array}$ \\
\hline $\begin{array}{c}\text { Study Group } \\
\text { Control }\end{array}$ & 37 & $16(43.2 \%)$ & $49.00 \pm 3.54$ & $8.05 \pm 1.37$ \\
Group & 33 & $14(42.4 \%)$ & $47.70 \pm 3.23$ & $8.18 \pm 1.51$ \\
$\chi^{2} / \mathrm{t}$ & - & 0.005 & 1.604 & 0.463 \\
$P$ & - & 0.945 & 0.113 & 0.643 \\
\hline
\end{tabular}


2) patients with subarachnoid hemorrhage from other causes; 3 ) pregnant and lactating women; 4) patients with postoperative secondary hydrocephalus, intracranial infection, and rebleeding; 5) patients who were moribund, died, or had treatment interruptions on admission; 6) patients with ruptured aneurysms massive bleeding resulted from surgery or aggravated CVS caused by failed embolization; 7) poor patient and family compliance $(<80 \%,>120 \%)$.

\subsection{Shedding Criteria}

1) Subjects who deteriorated or in whom a serious adverse event may/have occurred during the course of the study should be discontinued from this study based on physicians' judgment. Patients whose condition deteriorated were regarded as invalid cases; 2) subjects in whom certain pathological changes occurred or who combined or complicated with other diseases during the course of the study should be discontinued from this study based on physicians' judgment; 3) subjects who were unwilling to continue during the course of study and requesting withdrawal.

\subsection{Therapeutic Regime}

Test drugs: Nimodipine Injection $(10 \mathrm{mg}, 50 \mathrm{ml})$ was from Bayer, Germany, and Shenmai injection $(100 \mathrm{ml})$ was from Zhengda adolescentis Bao pharmaceutical company. The aneurysm was clipped, contoured or wrapped by craniotomy in all 60 cases, and the patients were generally treated by routine therapy including respiratory support, ECG monitoring, maintenance of perfusion pressure, prevention of cerebral edema, control of intracranial pressure, neurotrophy, correction of metabolic imbalance, prevention of stress ulcers, and antibiotic prophylaxis against infection if necessary. Study group: patients were given a continuous pump of nimodipine immediately after surgery. A starting dose of 0.5 $\mathrm{mg} / \mathrm{h}$ is given to patients who weigh less than $70 \mathrm{~kg}$ or have unstable blood pressure, which can be increased to $1 \mathrm{mg} / \mathrm{h}$ after $2 \mathrm{~h}$; patients weighing $70 \mathrm{~kg}$ and above were started at $1 \mathrm{mg} / \mathrm{h}$, and the dose could be up to $2 \mathrm{mg} / \mathrm{h}$ after $2 \mathrm{~h}$. Thereafter additional doses were applied depending on the extent of CVS for different patients for $14 \mathrm{~d}$. At the same time, $100 \mathrm{ml}$ Shenmai injection diluted with $250 \mathrm{ml}$ of $5 \%$ glucose was intravenously instilled once daily (diabetes patients were given insulin polarization), and the instillation rate was controlled to be within $40 \mathrm{drops} / \mathrm{min}$. Control group: on the basis of routine treatment with nimodipine the same as the study group, $350 \mathrm{ml}$ intravenous infusion with $5 \%$ glucose was added once a day.

\subsection{Observations}

1) TCD was used to detect blood flow velocity $(\mathrm{Vm})$ of the MCA in the affected side at $1,3,7,11$ and 14 days after the treatment of ruptured aneurysms in both groups; 2) the incidence of CVS at 1, 3, 7, 11 and 14 days after surgery was observed in both groups, and the number of new cerebral infarctions was counted by 
cranial CT/MRI; 3) the Glasgow Coma Scale (GCS) scores and Glasgow Outcome Scale (GOS) scores were respectively used to assess the status of patients at 14 days postoperatively and the prognosis of patients at 6 months postoperatively in both groups.

\subsection{Statistical Methods}

Statistical analyses were performed with SPSS 22.0. Measurement data were described as mean differences \pm standard deviation $(\bar{X} \pm s)$, conformed to normal distribution using independent samples t-test for comparison between two groups, and non-normal distribution using rank-sum test. Count data were expressed by rate, using $\chi^{2}$ test. Test level $\mathrm{a}=0.05$.

\section{Result}

As shown in Table 2, there were no statistically significant differences between the two groups in the mean blood flow velocity $(\mathrm{Vm})$ of the ipsilateral middle cerebral artery on postoperative days $1,3,7,11$, or $14(P>0.05)$. Besides, as shown in Table 3, there were no statistically significant differences between the two groups in the incidence of cerebral vasospasm at 1,3,7,11, and 14 days after surgery $(P>0.05)$. However, the peak of $\mathrm{Vm}$ and the occurrence of cerebral vasospasm in the study group were earlier than those in the control group, and the cycle length of cerebral vasospasm occurrence was shortened (Figure 1). As shown in Table 4, there were no statistically significant differences in the GCS and GOS scores between the two groups after treatment $(P>0.05)$.

Table 2. Comparison of Vm during treatment between the two groups $(\mathrm{cm} / \mathrm{s})$.

\begin{tabular}{cccccc}
\hline Group & $\begin{array}{c}\text { Vm on } \\
\text { day } 1\end{array}$ & Vm on day 3 & Vm on day 7 & $\begin{array}{c}\text { Vm on } \\
\text { day } 11\end{array}$ & Vm on day 14 \\
\hline Study Group & $94.21 \pm 13.61$ & $106.82 \pm 11.55$ & $118.41 \pm 15.22$ & $113.92 \pm 12.81$ & $93.47 \pm 13.06$ \\
$\begin{array}{c}\text { Control } \\
\text { Group }\end{array}$ & $97.52 \pm 16.76$ & $105.46 \pm 13.09$ & $115.70 \pm 12.06$ & $119.05 \pm 14.52$ & $94.34 \pm 15.30$ \\
$\mathrm{t}$ & 0.914 & 0.463 & 0.888 & 1.571 & 0.256 \\
$P$ & 0.364 & 0.645 & 0.374 & 0.121 & 0.799 \\
\hline
\end{tabular}

Table 3. Occurrence of cerebral vasospasm during treatment in two groups.

\begin{tabular}{cccccc}
\hline Group & $\begin{array}{c}\text { Number of } \\
\text { CVS on day 1 }\end{array}$ & $\begin{array}{c}\text { Number of } \\
\text { CVS on day 3 }\end{array}$ & $\begin{array}{c}\text { Number of } \\
\text { CVS on day 7 }\end{array}$ & $\begin{array}{c}\text { Number of } \\
\text { CVS on day 11 }\end{array}$ & $\begin{array}{c}\text { Number of } \\
\text { CVS on day 14 }\end{array}$ \\
\hline $\begin{array}{c}\text { Study Group } \\
\text { Control }\end{array}$ & $2(5.4 \%)$ & $5(13.5 \%)$ & $13(35.1 \%)$ & $10(27.0 \%)$ & $1(2.7 \%)$ \\
Group & $4(12.1 \%)$ & $4(12.1 \%)$ & $9(27.3 \%)$ & $13(39.4 \%)$ & $0(0.0 \%)$ \\
$\chi^{2}$ & 0.330 & 0.030 & 0.500 & 1.209 & 1.288 \\
$P$ & 0.566 & 0.862 & 0.479 & 0.271 & 0.256 \\
\hline
\end{tabular}


Table 4. Comparison of GCS and GOS scores after treatment between the two groups.

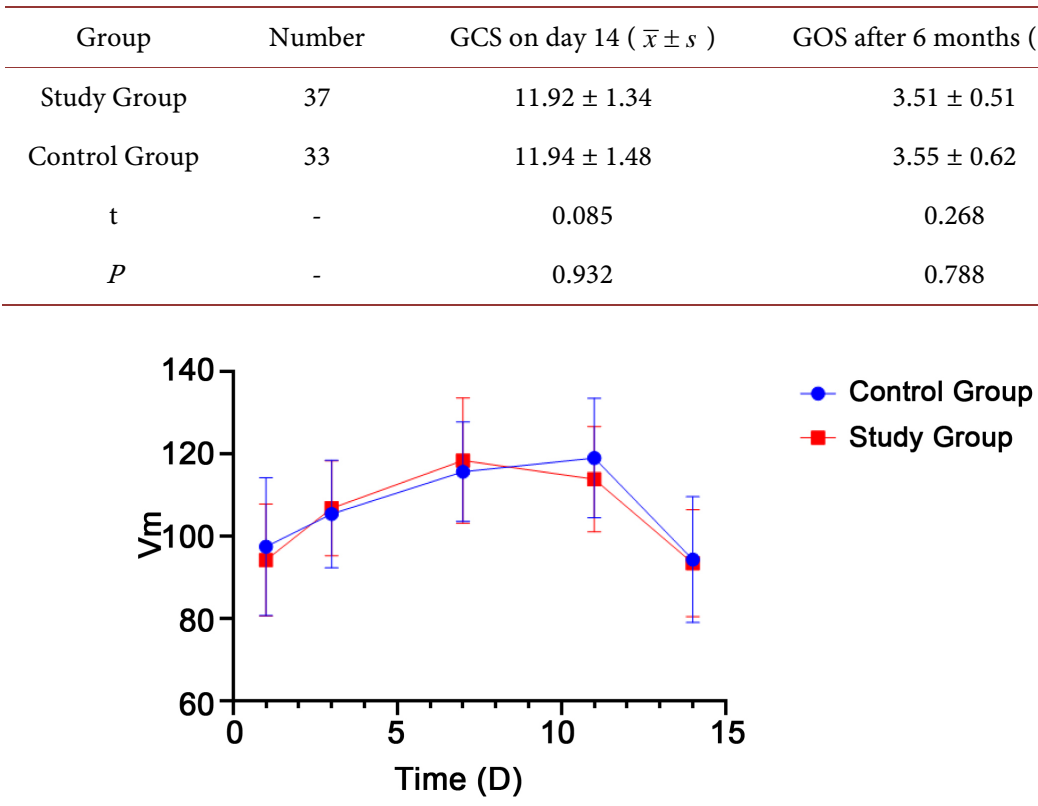

Figure 1. Dynamic changes in Vm during treatment in the two groups.

\section{Discussion}

Aneurysmal subarachnoid hemorrhage (aSAH) damages not only the brain but also multiple organ systems, and most patients have a poor prognosis [12]. In recent years, the promoted application of neurosurgical aneurysm clipping surgery and endovascular intervention has greatly reduced the mortality and disability rates of patients with ruptured cerebral aneurysms, but postoperative cerebral vasospasm (CVS) still remains a great difficulty in clinical treatment [13].

Nearly $70 \%-90 \%$ of patients with aSAH will develop cerebral vasospasm, of which approximately $70 \%$ are transient, early-onset constrictions of vessels triggered by mechanical stimuli, termed angiographic cerebral vasospasm or asymptomatic vasospasm; about $30 \%$ are late-onset cerebral vasospasm or symptomatic vasospasm, which usually occurs 3 - 13 days after aSAH and is most commonly observed on postoperative day 8 . The patients are accompanied by ischemic neurological dysfunction, high disability rate and mortality, and need aggressive intervention [14] [15]. The main mechanisms of delayed cerebral vasospasm are as following: 1) the imbalance between endothelial relaxing factors dominated by nitric oxide (NO) and vasoconstriction factors represented by endothelin-1 (ET-1), as well as the blockade of calcium channels, lead to reversible vasoconstriction [16] [17] [18]; 2) a large number of immune inflammatory mediators are released in a cascade, upregulating the expression of cytokines such as C-reactive protein, monocyte chemokine, tumor necrosis factor (TNF- $\alpha$ ), IL-1, IL-6, and IL-8, accompanied by neutrophil activation [19] [20] [21] [22]; 3) oxyhemoglobin released from erythrocyte degradation in aSAH leads to vasospasm by directly stimulating smooth muscle to contract, releasing vasoactive substances, producing lipid peroxides, and so on [15] [23] [24]; 4) 
gene expression and apoptosis: apoptotic cells are present in the intima of arteries of aSAH patients, and inhibition of endothelial cell apoptosis by recombinant human erythropoietin or p53 inhibitors prevents vasospasm [25] [26]. All of the above factors can lead to the disturbance of ion pump function, intracellular calcium overload, protein kinase $\mathrm{C}$ activation, inflammatory reaction and cell proliferation in vascular smooth muscle cells, and finally promote the abnormal contraction of vascular smooth muscle to cause CVS. Nimodipine, a dihydropyridine L-type calcium channel antagonist that inhibits calcium influx in intracranial vascular smooth muscle cells, has been a conventional treatment for cerebral vasospasm after aSAH due to its effects of alleviating vasospasm, reducing microthrombosis, improving function of ischemic brain tissues, and neuroprotection [27]. However, the vasomotor effects of nimodipine can predispose unstable haemodynamics patients to adverse effects such as hypotension, and avoid more severe neurological damage from hypoperfusion, some patients are unable to complete the full course of treatment, resulting in poor outcomes [28].

In China, subarachnoid hemorrhage is classified as "stroke" in traditional Chinese medicine science, and the location of the disease is in the brain. It is common in the elderly with weakness due to senility, intestinal obstruction and deficiency of liver-yin and kidney-yin, which can lead to deficiency of viscera, stagnation of Qi, imbalance of yin and Yang, and pathogenic phlegm-heat over time. Phlegm and heat complication generates wind; wind forces blood to move; disorder of gas-blood invades the brain; blood overflows outside the veins, causing damage to the spiritual mechanism [29]. Acute subarachnoid hemorrhage is mainly manifested by the phlegm-heat excess syndrome, and according to the principles of treating the incidental aspect of disease at acute phase and treating the root cause in recovery period, treatment is based on clearing heat-fire, activating blood and eliminating stasis, washing phlegm and inducing diuresis, and viscera-unblocking and resuscitation [30].

Shenmai injection, derived from ancient prescriptions, is mainly extracted from red ginseng and ancient prescriptions, and modern pharmacology has confirmed that its active ingredients are ginsenosides, arginine glycosides, ophiopogon japonicus saponin, polysaccharides, which have the efficacy of activating blood circulation, supplementing qi, nourishing yin and generating body fluid. Its main pharmacological mechanisms include the following: 1) promoting ATP synthesis, enhancing cardiac contractility, increasing cardiac output and mean arterial pressure, and guaranteeing blood supply to ischemic tissues [31];2) bidirectionally regulating blood pressure and improving microcirculation [32] [33]; 3) scavenging oxygenderived free radicals of brain tissue, inhibiting lipid peroxidation, enhancing SOD activity and stabilizing cell membrane, thus alleviating nerve cell ischemic injury, increasing the ability of cells to tolerate hypoxia, rescuing nerve cells with secondary ischemic injury to promote the recovery of neurological function and protect against cerebral ischemia-reperfusion injury [1]; 4) inhibiting immune inflammatory response and reducing cytokine levels 
to attenuate cellular inflammatory injury and protect vascular endothelial cells; 5) alleviating vasospasm by dilating blood vessels and anti platelet coacervation, thus achieving the protective effect of brain injury [34]. Studies have shown that Shenmai injection can restore ET-1/NO system homeostasis and increase the circulating arterial pressure to increase the perfusion level of brain tissue. Its efficacy in combination with nimodipine for CVS after traumatic SAH is exact [35]. Therefore, based on previous clinical experience and basic research work, this study chose a combination of Shenmai injection and nimodipine to prevent postoperative cerebral vasospasm from ruptured aneurysms, aiming to further seek a new scheme of integrated traditional Chinese and Western medicine that is based on conventional treatment while stabilizing arterial pressure in the circulatory system to guarantee an effective therapeutic dose of nimodipine. The results showed that patients treated with Shenmai injection combined with nimodipine had earlier peak blood flow velocity in the middle cerebral artery and shorter CVS cycles than the control group, and had neither significant improvement in the incidence of CVS nor in the GCS score but earlier CVS recovery time. The peak value of VM in the control group appeared in advance and the occurrence cycle of CVS was relatively shortened. These results suggested that Shenmai injection may ameliorate blood vessel spasm more quickly by improving hemodynamics to guarantee the dosage and efficacy of nimodipine use and alleviate the adverse effects resulting from the long-term use of the drug, thus exerting a cerebral protective effect.

In conclusion, the results of this experiment demonstrated that compared with nimodipine alone, Shenmai injection combined with nimodipine can shorten the occurrence cycle of CVS without affecting the efficacy of nimodipine in preventing CVS. However, the sample size of this study was small; the observational indexes were not comprehensive; and the exact efficacy of Shenmai injection for preventing and treating CVS after aSAH was not observed. It is possible that CVS is only one of the critical factors affecting the prognosis of intracerebral hemorrhage after aneurysm rupture, while the prognosis is closely related to other factors such as hemorrhage size and complications [31] [36]. More research on the mechanism of action and clinical application of Shenmai injection on improving CVS after aSAH need to be conducted in the future.

\section{Authors' Contributions}

Tianya $\mathrm{Wu}$ and Jingxin $\mathrm{Fu}$, as the main writers of this study, contributed equally. They completed the methodalogy and investigation and wrote the main part of the manuscript. Qichao Chen took part in the the data curation and formal analysis. Longbiao $\mathrm{Xu}$, as the director and supervisor of the project, administrated the report writing. All authors read and agree to the final text.

\section{Fund Projects}

This study was funded by Clinical research project of Zhejiang Medical Associa- 
tion (No. 2019ZYC-A52); Zhejiang Medical and Health Science and Technology (2020PY081).

\section{Conflicts of Interest}

The authors declare no conflicts of interest regarding the publication of this paper.

\section{References}

[1] Feigin, V.L., Lawes, C.M., Bennett, D.A., Barker-Collo, S.L. and Parag, V. (2009) Worldwide Stroke Incidence and Early Case Fatality Reported in 56 Population-Based Studies: A Systematic Review. The Lancet Neurology, 8, 355-369.

https://doi.org/10.1016/S1474-4422(09)70025-0

[2] Kundra, S., Mahendru, V., Gupta, V. and Choudhary, A.K. (2014) Principles of Neuroanesthesia in Aneurysmal Subarachnoid Hemorrhage. Journal of Anaesthesiology, Clinical Pharmacology, 30, 328-337. https://doi.org/10.4103/0970-9185.137261

[3] Passier, P.E., Visser-Meily, J.M., van Zandvoort, M.J., Post, M.W., Rinkel, G.J. and van Heugten, C. (2010) Prevalence and Determinants of Cognitive Complaints after Aneurysmal Subarachnoid Hemorrhage. Cerebrovascular Diseases, 29, 557-563. https://doi.org/10.1159/000306642

[4] Mocco, J., Brown, R.D., Torner, J.C., Capuano, A.W., Fargen, K.M., Raghavan, M.L., et al. (2018) Aneurysm Morphology and Prediction of Rupture: An International Study of Unruptured Intracranial Aneurysms Analysis. Neurosurgery, 82, 491-496. https://doi.org/10.1093/neuros/nyx226

[5] Sweeting, M.J., Ulug, P., Powell, J.T., Desgranges, P., Balm, R. and Ruptured Aneurysm, T. (2015) Ruptured Aneurysm Trials: The Importance of Longer-Term Outcomes and Meta-Analysis for 1-Year Mortality. European Journal of Vascular and Endovascular Surgery, 50, 297-302. https://doi.org/10.1016/j.ejvs.2015.04.015

[6] Spetzler, R.F., McDougall, C.G., Zabramski, J.M., Albuquerque, F.C., Hills, N.K., Russin, J.J., et al. (2015) The Barrow Ruptured Aneurysm Trial: 6-Year Results. Journal of Neurosurgery, 123, 609-617. https://doi.org/10.3171/2014.9.JNS141749

[7] Feigin, V.L., Rinkel, G.J., Algra, A., Vermeulen, M. and van Gijn, J. (1998) Calcium Antagonists in Patients with Aneurysmal Subarachnoid Hemorrhage: A Systematic Review. Neurology, 50, 876-883. https://doi.org/10.1212/WNL.50.4.876

[8] Bele, S., Proescholdt, M.A., Hochreiter, A., Schuierer, G., Scheitzach, J., Wendl, C., et al. (2015) Continuous Intra-Arterial Nimodipine Infusion in Patients with Severe Refractory Cerebral Vasospasm after Aneurysmal Subarachnoid Hemorrhage: A Feasibility Study and Outcome Results. Acta Neurochirurgica, 157, 2041-2050. https://doi.org/10.1007/s00701-015-2597-z

[9] Bath, P.M. and Krishnan, K. (2014) Interventions for Deliberately Altering Blood Pressure in Acute Stroke. Cochrane Database of Systematic Reviews, No. 3, CD000039. https://doi.org/10.1002/14651858.CD000039.pub3

[10] Li, J.S., Wang, H.F., Li, S.Y., Yu, X.Q. and Wang, Z.W. (2011) Shenmai Injection for Chronic Pulmonary Heart Disease: A Systematic Review and Meta-Analysis. Journal of Alternative and Complementary Medicine, 17, 579-587.

https://doi.org/10.1089/acm.2010.0354

[11] Saqqur, M., Khan, K., Derksen, C., Alexandrov, A. and Shuaib, A. (2018) Transcranial Doppler and Transcranial Color Duplex in Defining Collateral Cerebral 
Blood Flow. Journal of Neuroimaging, 28, 455-476.

https://doi.org/10.1111/jon.12535

[12] Petridis, A.K., Kamp, M.A., Cornelius, J.F., Beez, T., Beseoglu, K., Turowski, B., et al. (2017) Aneurysmal Subarachnoid Hemorrhage. Deutsches Ärzteblatt International, 114, 226-236. https://doi.org/10.3238/arztebl.2017.0226

[13] Datar, S. and Rabinstein, A.A. (2017) Postinterventional Critical Care Management of Aneurysmal Subarachnoid Hemorrhage. Current Opinion in Critical Care, 23, 87-93. https://doi.org/10.1097/MCC.0000000000000391

[14] Suhardja, A. (2004) Mechanisms of Disease: Roles of Nitric Oxide and Endothelin-1 in Delayed Cerebral Vasospasm Produced by Aneurysmal Subarachnoid Hemorrhage. Nature Clinical Practice: Cardiovascular Medicine, 1, 110-116. https://doi.org/10.1038/ncpcardio0046

[15] Oyama, K. and Criddle, L. (2004) Vasospasm after Aneurysmal Subarachnoid Hemorrhage. Critical Care Nurse, 24, 58-60, 62, 64-57.

https://doi.org/10.4037/ccn2004.24.5.58

[16] Lin, C.L., Jeng, A.Y., Howng, S.L. and Kwan, A.L. (2004) Endothelin and Subarachnoid Hemorrhage-Induced Cerebral Vasospasm: Pathogenesis and Treatment. Current Medicinal Chemistry, 11, 1779-1791. https://doi.org/10.2174/0929867043364919

[17] Ng, W.H., Moochhala, S., Yeo, T.T., Ong, P.L. and Ng, P.Y. (2001) Nitric Oxide and Subarachnoid Hemorrhage: Elevated Level in Cerebrospinal Fluid and Their Implications. Neurosurgery, 49, 622-626. https://doi.org/10.1227/00006123-200109000-00016

[18] Allen, G.S. (1985) Role of Calcium Antagonists in Cerebral Arterial Spasm. American Journal of Cardiology, 55, 149B-153B. https://doi.org/10.1016/0002-9149(85)90624-1

[19] Lucke-Wold, B.P., Logsdon, A.F., Manoranjan, B., Turner, R.C., McConnell, E., Vates, G.E., et al. (2016) Aneurysmal Subarachnoid Hemorrhage and Neuroinflammation: A Comprehensive Review. International Journal of Molecular Sciences, 17, 497. https://doi.org/10.3390/ijms17040497

[20] Inagawa, T. (2016) Risk Factors for Cerebral Vasospasm Following Aneurysmal Subarachnoid Hemorrhage: A Review of the Literature. World Neurosurgery, 85, 56-76. https://doi.org/10.1016/j.wneu.2015.08.052

[21] Provencio, J.J., Fu, X., Siu, A., Rasmussen, P.A., Hazen, S.L. and Ransohoff, R.M. (2010) CSF Neutrophils Are Implicated in the Development of Vasospasm in Subarachnoid Hemorrhage. Neurocritical Care, 12, 244-251. https://doi.org/10.1007/s12028-009-9308-7

[22] Provencio, J.J. and Vora, N. (2005) Subarachnoid Hemorrhage and Inflammation: Bench to Bedside and Back. Seminars in Neurology, 25, 435-444.

https://doi.org/10.1055/s-2005-923537

[23] Leclerc, J.L., Blackburn, S., Neal, D., Mendez, N.V., Wharton, J.A., Waters, M.F., et al. (2015) Haptoglobin Phenotype Predicts the Development of Focal and Global Cerebral Vasospasm and May Influence Outcomes after Aneurysmal Subarachnoid Hemorrhage. Proceedings of the National Academy of Sciences of the United States of America, 112, 1155-1160. https://doi.org/10.1073/pnas.1412833112

[24] Macdonald, R.L. and Weir, B.K. (1991) A Review of Hemoglobin and the Pathogenesis of Cerebral Vasospasm. Stroke, 22, 971-982.

https://doi.org/10.1161/01.STR.22.8.971

[25] Zhou, C., Yamaguchi, M., Colohan, A.R. and Zhang, J.H. (2005) Role of p53 and 
Apoptosis in Cerebral Vasospasm after Experimental Subarachnoid Hemorrhage. Journal of Cerebral Blood Flow and Metabolism, 25, 572-582. https://doi.org/10.1038/sj.jcbfm.9600069

[26] Carlson, A.P., Hanggi, D., Macdonald, R.L. and Shuttleworth, C.W. (2020) Nimodipine Reappraised: An Old Drug with a Future. Current Neuropharmacology, 18, 65-82. https://doi.org/10.2174/1570159X17666190927113021

[27] Hajizadeh Barfejani, A., Rabinstein, A.A., Wijdicks, E.F.M. and Clark, S.L. (2019) Poor Utilization of Nimodipine in Aneurysmal Subarachnoid Hemorrhage. Journal of Stroke and Cerebrovascular Diseases, 28, 2155-2158. https://doi.org/10.1016/j.jstrokecerebrovasdis.2019.04.024

[28] Wang, C., Zhao, X., Mao, S., Wang, Y., Cui, X. and Pu, Y. (2006) Management of SAH with Traditional Chinese Medicine in China. Neurological Research, 28, 436-444. https://doi.org/10.1179/016164106X115044

[29] Li, Y., Li, D., Tan, X. and Lu, C. (2015) Hemorrhagic Stroke Treated with Traditional Chinese Medicine: A Review. Hunan Journal of Traditional Chinese Medicine, 31, 174-176.

[30] Ciurea, A.V., Palade, C., Voinescu, D. and Nica, D.A. (2013) Subarachnoid Hemorrhage and Cerebral Vasospasm-Literature Review. Journal of Medicine and Life, 6, 120-125.

[31] Shi, L., Wang, Y., Zhang, J. and Zuo, F. (2010) Shenmai Injection in the Treatment of Subarachnoid Hemorrhage. Journal of Chongqing Medical University, 35, 1411-1413.

[32] Shi, L., Xie, Y., Liao, X., Chai, Y. and Luo, Y. (2015) Shenmai Injection as an Adjuvant Treatment for Chronic Cor Pulmonale Heart Failure: A Systematic Review and Meta-Analysis of Randomized Controlled Trials. BMC Complementary and Alternative Medicine, 15, 418. https://doi.org/10.1186/s12906-015-0939-2

[33] He, J., Lu, F., Qin, G., et al. (2020) Observation on the Efficacy of Shenmai Injection Combined with Milrinone in the Treatment of Refractory Heart Failure. Chinese Journal of Traditional Chinese Medicine, 38, 203-206.

[34] Shen, U., Lu, G., Cai, Z. and Xue, Z. (2019) Effect of Shenmai Injection on Antioxidative Effect and Serum Levels of IL-6, IL-12, and TNF- $\alpha$ in Patients with Severe Alcoholic Hepatitis. Chinese Archives of Traditional Chinese Medicine, 37, 2554-2557.

[35] Ying, Y., Xu, X. and Ye, R. (2019) Effect of Shenmai Injection Combined with Nimodipine in Traumatic Subarachnoid Hemorrhage Patients with Cerebral Vasospasm. Zhejiang Medical Journal, 41, 457-461.

[36] Zhao, Q., Gou, W. and Ma, Y. (2012) Shenmai Injection in Cerebral Vasospasm after Subarachnoid Hemorrhage. Chinese Community Doctors, 14, 199-200. 